\title{
The Correlation between Solar Flare Phenomena in an X-ray Region and Radio Flux Measurement from January to September 2010
}

\author{
Z. S. Hamidi ${ }^{1, *}$, N. N. M. Shariff ${ }^{2}$, M. F. Ali ${ }^{1}$, C. Monstein ${ }^{3}$, W. N. A. Wan Zulkifli ${ }^{1}$, \\ M. B. Ibrahim ${ }^{1}$, N. S. Arifin ${ }^{1}$, N. A. Amran ${ }^{1}$ \\ ${ }^{1}$ School of Physics and Material Sciences, Faculty of Sciences, MARA University of Technology, \\ 40450, Shah Alam, Selangor, Malaysia \\ ${ }^{2}$ Academy of Contemporary Islamic Studies (ACIS), MARA University of Technology, \\ 40450, Shah Alam, Selangor, Malaysia \\ ${ }^{3}$ Institute of Astronomy, Wolfgang-Pauli-Strasse 27, Building HIT, Floor J, \\ $\mathrm{CH}-8093$ Zurich, Switzerland \\ *E-mail address: zetysh@salam.uitm.edu.my
}

\begin{abstract}
A short term variation of solar flare in nine months (January 2010 to September 2010) is presented. This paper review and analyze the correlation between radio flux strength measurement and solar flare in the X-ray region. The radio flux measurement data were taken from the National Research Council; Ottawa while hard X-ray emission observed by Royal Observatory of Belgium. The overall range of solar radio flux recorded in this study ranging from $68 \times 10^{-22} \mathrm{Wm}^{-2} \mathrm{~Hz}^{-1}$ to $96 \times 10^{-22}$ $\mathrm{Wm}^{-2} \mathrm{~Hz}^{-1}$. As there was no class of an $\mathrm{X}$ of solar flare reported at all in this study, we can confirm that there are no major effects that happened on Earth and outer space such as Coronal Mass Ejections (CMEs) and solar storms. We concluded that the Sun shows a very minimum activity towards $24^{\text {th }}$ solar cycle.
\end{abstract}

Keywords: Sun; chromospheres; flares; radio flux; solar activity

\section{INTRODUCTION}

The Sun generates solar flares and Coronal Mass Ejections (CMEs) events in an approximate 11-year cycle. Solar flares, a sudden large energy release $\left(10^{29} \mathrm{erg}\right.$ in $\left.10^{2-3} \mathrm{~s}\right)$ in a magnetic active region that take place in the outermost layer of the corona are the most active manifestation of solar activity and more importantly for solar radio astronomy. This phenomenon has been observed by Carrington in 1859, the first observation in white light. It can occur everywhere on the Sun, in active regions, penumbras, on the boundaries of the magnetic network of the quiet Sun, and even in the network interior [1,2]. Theoretically, the impulsiveness of the flare energy release is possibly triggered by a sudden change in resistivity of high-frequency (collision less) wave turbulence and is probably related to the capability of particle acceleration. It can be observed at all wavelengths from decameter radio waves to the gamma-rays at $100 \mathrm{MeV}$. This phenomenon is classified according to the order 
of magnitude of the peak burst intensity measured at the Earth in the 0.1 to $0.8 \mathrm{~nm}$ wavelength band. There are five types $\mathrm{A}, \mathrm{B}, \mathrm{C}, \mathrm{M}$ and $\mathrm{X}$ where $\mathrm{X}$ is the largest type comparing others. In certain cases, the rare with very strong flare events also show the emission in the integrated light. During the period of high activity, solar flares are relatively frequent (more than 1 to 2 per hour at maximum). It also can be classified by different importance groups which are $\mathrm{X}, \mathrm{M}$ and $\mathrm{C}$ classes according to their area and brightness in the $\mathrm{X}$-ray region [3].

Solar flares in radio region covers from millimeter to meter wavelengths is expected to have two main characteristics. First, since optically-thin thermal emission has a flat flux spectrum, it should become increasingly important relative to the falling spectrum of nonthermal gyrosynchrotron emission as one goes to higher frequencies [4]. Secondly, higher-frequency nonthermal gyrosynchrotron emission requires emission at ever higher harmonics of the electron gyrofrequency, and this makes it sensitive to electrons with much higher energies than are required for microwave emission $(\mathrm{MeV}$ and higher energies rather than tens to hundreds of $\mathrm{keV}$ [5]. It is believed that the change of magnetic field lines potentially creates the solar flares. Usually, flares emit bursts of this region from 1-60 $\mathrm{m}$ are observed as sudden and short-lived brightening of plage region in the neighborhood of sunspots. There are several investigations of the types of radio bursts in the range $0.1-4 \mathrm{GHz}$ that occur in flares that exhibit hard X-rays, using data from the Phoenix-2 spectrometer operated by ETH-Zürich [6]. Normally, the flare radio spectra peak at $10 \mathrm{GHz}$, although in larger flares the spectral peak can often occur at much higher frequencies [7]. Meanwhile, complementary observations of energetic electrons produced by the gyrosynchrotron radiation in radio emission (centimeter/millimeter wavelengths) are important to observe the flux that produced from the low corona of the Sun. These radio fluxes are produced by electron beams along or closed field lines which can also be classified as a radio type of III, J, U, N and RS bursts [8].

On the other hand, X-ray spikes appear to result mainly from high energy electrons which produce a significant Bremsstrahlung contribution at lower energies [9]. The first observation of a flare at hard X-ray $/ \gamma$-ray wavelengths was carried out with a balloon-borne telescope over Cuba [10]. In this region, the electrons that produce hard X-ray emission have energies of order $10 \mathrm{keV}$ or more, and such energetic electrons are also very efficient emitters of radio emission in the solar corona [11]. The study of hard X-ray emission from the Sun has had a naturally in solar radio emission [12]. It is well known that there is generally a discrepancy between the energy distribution of the non-thermal electrons that produce the HXRs and those that produce the radio emission at high frequencies: the radio data generally show a harder spectrum $[13,14]$.

The first solar flares towards 24th Solar Cycle have been detected in 4th of January 2012 [15]. Luckily, it still has no dangerous effect on the GPS operations. In Malaysia, the first geomagnetic storm has been reported in March 2012 [16,17]. Due to date, last geomagnetic storm was reported to occur in the December 2006 for about 10-15 minutes, which is enough to cause disruption on the GPS signal transmission based on its high energy particles that collides with the earth magneto sphere. The flare problem is still one of the great puzzles of cosmic physics at our time. They usually are displayed in the most of the Fraunhofer lines, especially at $\mathrm{H} \alpha$ and $\mathrm{H}$ and $\mathrm{K}$ lines of Ca. Ideally, magnetic reconnection is believed to be the primary energy release mechanism, heat the plasma and accelerate particles high in the corona [18]. The released energy is transported downward along the newly reconnected closed flaring loop by non-thermal particles and/or thermally conductive, heating the chromospheric material rapidly (at a rate faster than the radiative and conductive cooling 
rates) up to a temperature of $\sim 10^{7} \mathrm{~K}$. As the result of the overpressure drives a mass flow upward along the loop at a speed of a few hundred $\mathrm{km} \mathrm{s}^{-1}$, which fills the flaring loop with hot plasma, giving rise to the gradual evolution of soft X-ray (SXR) emission [19].

Interestingly, combinations of X-ray and radio observations of the Sun provide powerful diagnostics of particle acceleration and transport effects during solar flares. However, times profiles at radio wavelength and X-ray are not always correlated. The main reason is that in the X-ray region, the data are taken from the space telescope while for radio region data is taken using a radio telescope which has a limitation in some aspects [20]. However, it could not be denied that radio observations are still important in terms of sensitivity of the line of-sight component of the coronal magnetic field. This method still be used to study problems associated with oscillations.

From hard X-ray and radio observations, it is found that electron acceleration occurs in a wide range of heights in the solar atmosphere. Moreover, electron energy spectra deduced at low and high energies from both hard X-ray and centimeter/millimeter observations will affect the correlation of both regions. For the next maximum in solar activity 2014-2015, it is believed that the solar activities will double maxima. There is why it is very important to monitor our Sun which has potentially reacted unusual phenomena. Based on that issue, we studied the Sun's radiative output and its interaction throughout the Earth's atmosphere remains at the highest priority scientific objective for understanding the sun-climate relationship [21].

The present work investigates the correlation between solar flare phenomena in the Xray and radio region in a nine month period starting from January 2010 till September 2010. We compile the data and analyses theoretically to observe the current activity of the Sun.

\section{II. DATA ANALYSIS}

One of the main reason, statistical analysis is important is to monitor the possibilities of the Sun is active. During the largest flares, it potentially hit the Earth's magnetic field and creating a magnetic storm that can be detected at points around the globe. Another effect is that the ionosphere itself can be disturbed, giving rise to an ionospheric storm. Consequently, this will degrade High Frequency (HF) communications and when particularly bad it can lead to a total HF blackout. In Malaysia, we also successfully observed the solar flares due to solar radio burst [15,22] and Coronal Mass Ejections (CMEs) [23,24]. Our advantage is that we can observe the Sun within 12 hours per day throughout a year [25]. Therefore, this study is very important to predict if there is an indicator that triggers the phenomena. This work focuses on recent observations in hard X-rays and radio waves. Therefore, the accuracy of the daily 10.7 $\mathrm{cm}$ solar flux measurements taken at the National Research Council, Ottawa, has been analyzed. Solar flux is used as the basic indicator of solar activity and to determine the level or radiation being received from the Sun. The solar flux is measured in Solar Flux Units (SFU) and is the amount of radio noise or flux that is emitted at a frequency of $2800 \mathrm{MHz}$ $(10.7 \mathrm{~cm})$. The measurements of the global solar radio flux are observed by Royal Observatory of Belgium during the impulsive phase in nine months in 2010 for the X-ray region by classifying the types of solar flare as in Table 1 . 


\section{RESULTS AND ANALYSIS}

For analysis and theoretical purposes, the data were periodically collected from the NOAA Space Weather Prediction Center (SWPC) website. Daily data have been compiled and analysis was made throughout the period of nine months. Table 1 shows the average value of solar flare based on different classes $(\mathrm{C}, \mathrm{M}$ and $\mathrm{X}$ ) classes and the $10.7 \mathrm{~cm}$ solar radio flux measurement.

The F10.7 is a daily index derived from solar radio measurements taken at $2800 \mathrm{MHz}$, excluding activity from solar flares. We analyzed the data early of January till end of September 2010.

Table 1. Average value of solar flares based on different classes (C, M and X) classes and the radio flux measurement.

\begin{tabular}{|c|c|c|c|c|c|}
\hline \multirow{2}{*}{ Months } & \multirow{2}{*}{$\begin{array}{c}\text { Solar Flares } \\
\text { Number }\end{array}$} & \multicolumn{3}{|c|}{ X-Ray } & \multirow{2}{*}{$\begin{array}{c}\text { Average } \\
\text { of } 10.7 \mathrm{~cm} \\
\text { solar radio } \\
\text { flux }\end{array}$} \\
\hline & & Class C & Class M & Class X & \\
\hline January & 34 & 28 & 6 & 0 & $11.3 \approx 11$ \\
\hline February & 53 & 44 & 9 & 0 & $17.7 \approx 18$ \\
\hline March & 9 & 9 & 0 & 0 & 3.0 \\
\hline April & 1 & 1 & 0 & 0 & $0.3 \approx 0$ \\
\hline May & 9 & 8 & 1 & 0 & 3.0 \\
\hline June & 11 & 9 & 2 & 0 & $3.7 \approx 4$ \\
\hline July & 10 & 10 & 0 & 0 & $3.3 \approx 3$ \\
\hline August & 9 & 8 & 1 & 0 & 3.0 \\
\hline September & 7 & 7 & 0 & 0 & $2.3 \approx 2$ \\
\hline Total & 143 & 124 & 19 & 0 & \\
\hline
\end{tabular}

The Sun was found to be most active in February with a total of 53 solar flares have been recorded. It is also shown that the radio flux obtained a highest peak on February where the Sun was found to be most active previously. This is proof that the dynamics of the acceleration region produced by sunspot in radio region has a linear correlation with hard-Xrays indicate an impulsive burst reconnection mode.

The level of flux measured at any given point above the Earth is dependent upon a number of factors, including the time of day, the season and most importantly is the phase of the sunspot cycle. It is found that the level of radiation from the Sun decreases as the number of solar activity decreases as in Figure 1. 


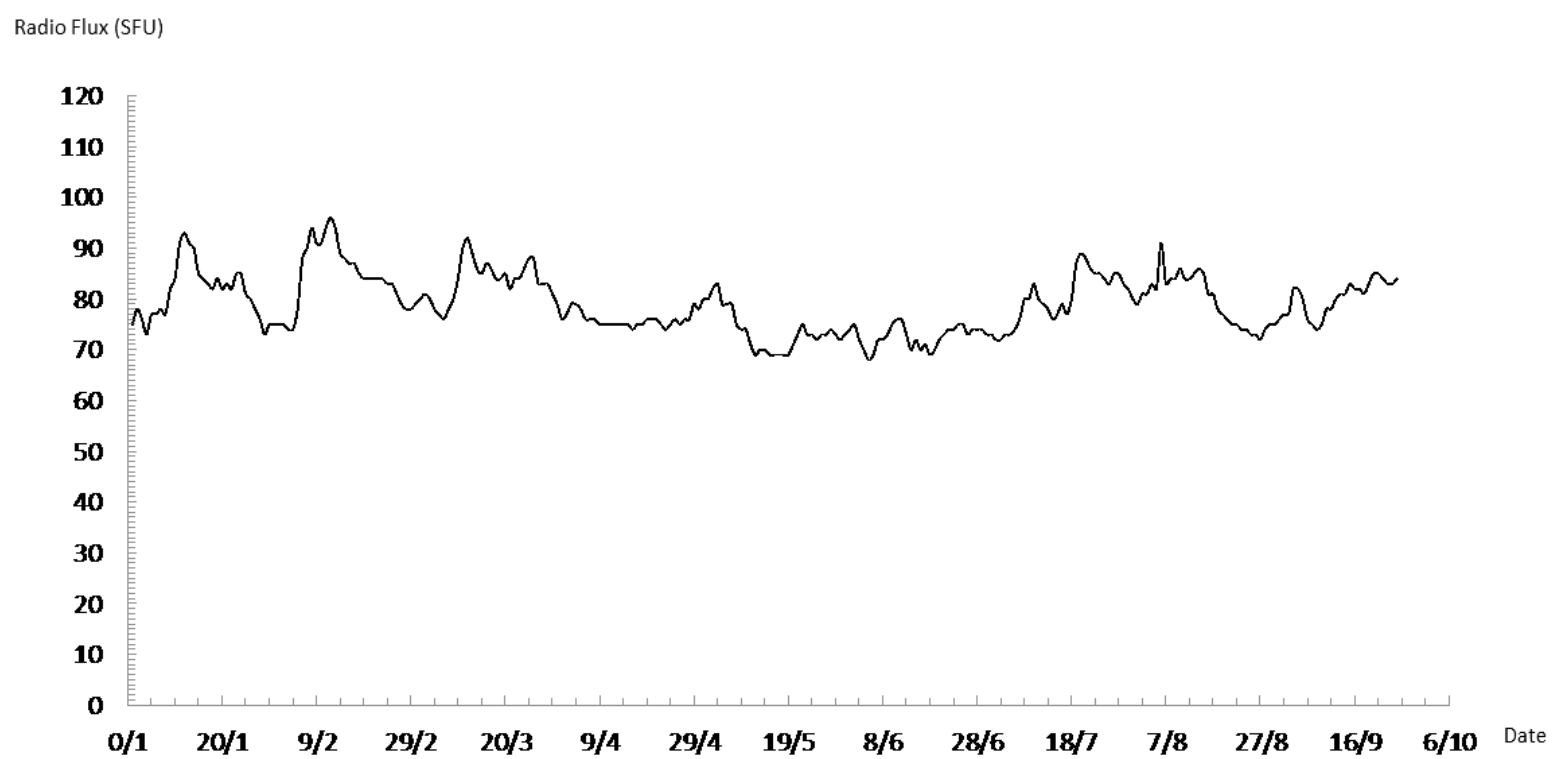

Figure 1. Pattern of radio flux $(10.7 \mathrm{~cm})$ within nine (9) months.

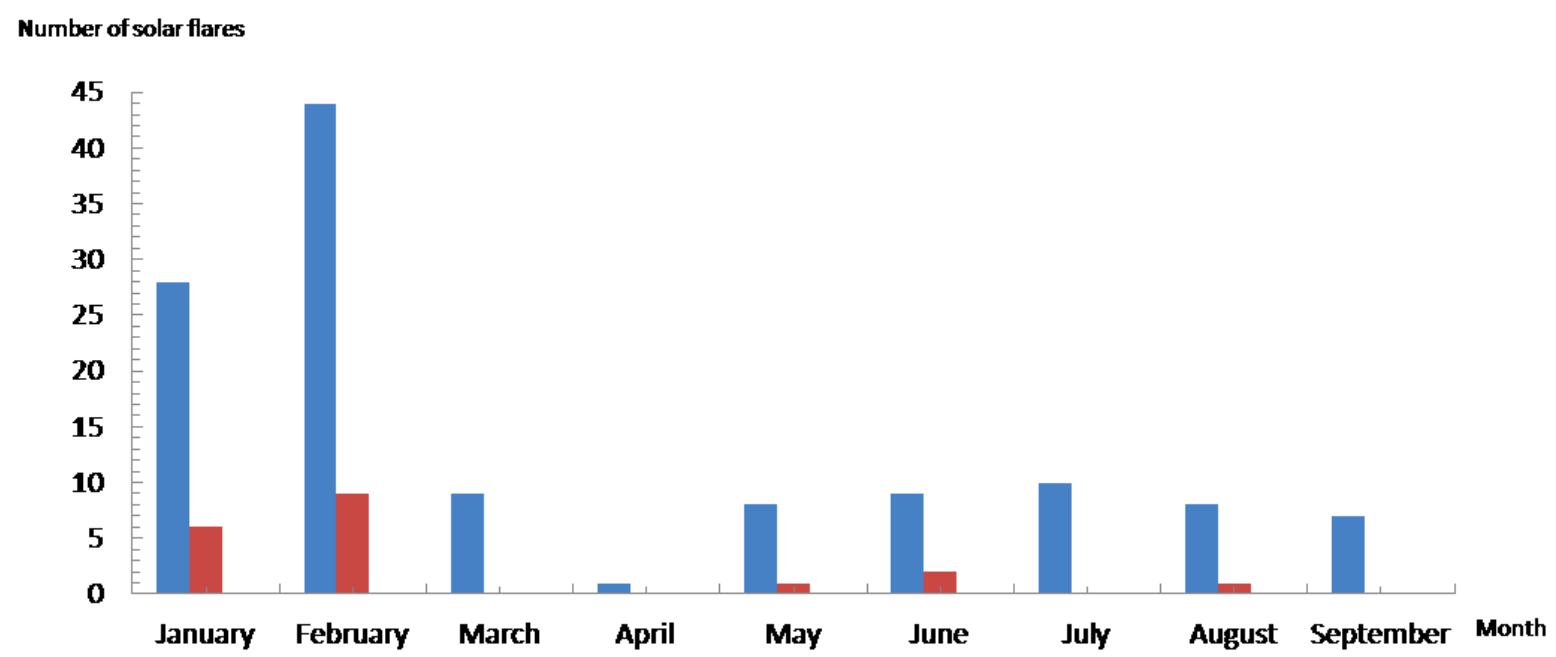

Figure 2. Distribution of types of solar flares of C and M class from January to September 2010. There is no X-class flare during that period.

Figure 2 shows the evolution of the flare at different energies, which divided into three (3) classes, C, M and X and radio flux at $10.7 \mathrm{~cm}$ observations. As there was no class $\mathrm{X}$ reported at all in this study, we can assume that there are no major effects that happened on earth and outer space such as CMEs and solar storms. These kinds of phenomena resulting from powerful solar flares corresponding to class X flares can actually affect people, but the possibilities are somehow restricted to several conditions. There exist a total number of 143 solar flares have been recorded and those are from $\mathrm{C}$ and M-class. Class $\mathrm{C}$ flares recorded the highest number which was 124 flares during the period of this study followed by class $\mathrm{M}$ 
flares which was 19 and lastly class X flares which were not detected at all. The average number of solar flares recorded in January, February, March, April, May, June, July, August and September was $11,18,3,0,3,4,3,3$ and 2 respectively.

In illustrating the relationship of solar radio flux and total number of solar flares $(\mathrm{C}, \mathrm{M}$ and $\mathrm{X}$ ) as shown in Figure 3, it can be explained by studying the range values of flux corresponding to flares values. From this case study, it was found that the minimum value of solar radio flux in order for the flares to occur is equivalent $68 \times 10^{-22} \mathrm{Wm}^{-2} \mathrm{~Hz}^{-1}$.

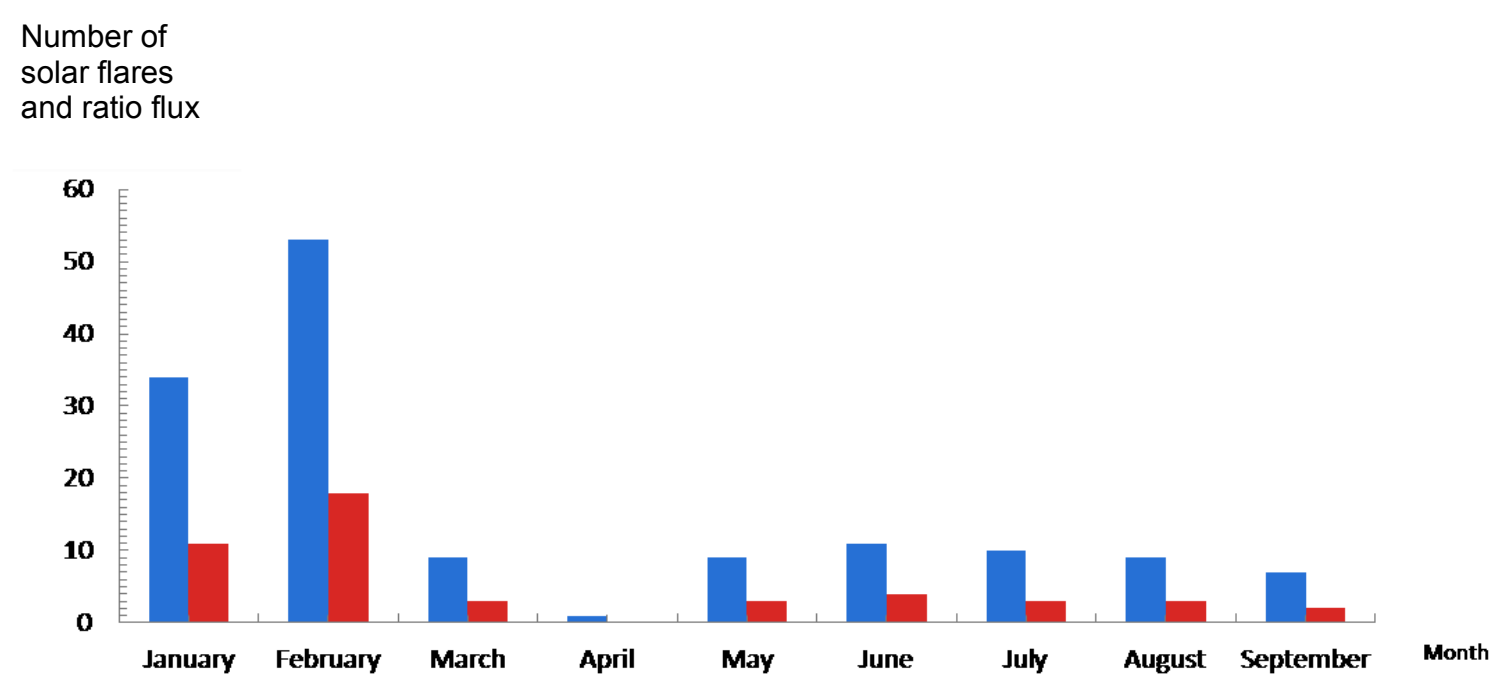

Figure 3. Correlation between the number of solar flares and an average of $10.7 \mathrm{~cm}$ radio flux from January to September 2010.

Thus, whenever the values of solar radio flux are high, there should be a higher number of flares produced by the Sun. The overall range of solar radio flux recorded in this study ranging from $68 \times 10^{-22} \mathrm{Wm}^{-2} \mathrm{~Hz}^{-1}$ to $96 \times 10^{-22} \mathrm{Wm}^{-2} \mathrm{~Hz}^{-1}$. This condition is in agreement with the relation as stated above and also supports that solar radio flux is closely related to flare production.

\section{CONCLUSIONS}

Results from this study have proven that solar flares and radio flux are quite closely related to the number of solar flares in an X-ray region. It could not be denied that the flare phenomena is not confined to thermal plasma, but includes high-energy particles and involves the corona. Therefore, the role of both regions is very important. It is confirmed Sun is in the quiet phase based on there is no X-class type of solar flare in this period. This is because the $\mathrm{X}$ class is the brightest classes amongst all. It is suggested that we need to analyze long term data in order to better understand the correlation between both regions. For the next maximum in solar activity 2014-2015, it is believed that the solar activities will double compared to current one. There is why it is very important to monitor the Sun which has potentially 
reacted an unusual phenomena. For overall, we concluded the Sun is still active as it keeps continuously emerging flares from time to time.

Still the flare process remains an enigma. Although the principle of magnetic reconnection is widely recognized to be the constituting element, the usual magneto hydrodynamic approach disregards that a large fraction of energy is released into accelerating electrons and ions. In this case, a non-thermal particles are the primary output of flares. In order to improve our understanding of the physical processes involved in particle acceleration in solar flares, numerical simulations of particle orbits have been conducted in international collaborations, we need to assume an alternative dissipative acceleration scenario. Our next focus is by monitoring the radio burst in the wider region to observe the pattern of burst and analyze critical aspect that might support this phenomenon.

\section{ACKNOWLEDGEMENT}

We are grateful to LASCO,SDO/AIA, NOAA and SWPC make their data available online. This work was partially supported by the FRGS (600 RMI/FRGS 5/3 2012) UiTM grants. Special thanks to the National Space Agency and the National Space Centre for giving us a site to set up this project and support this project. Solar burst monitoring is a project of cooperation between the Institute of Astronomy, ETH Zurich, and FHNW Windisch, Switzerland, MARA University of Technology and University of Malaya. This paper also used NOAA Space Weather Prediction Centre (SWPC) for the sunspot, radio flux and solar flare data for comparison purpose. The research has made use of the National Space Centre Facility and a part of an initiative of the International Space Weather Initiative (ISWI) program.

\section{BIOGRAPHY}

Zety Sharizat Hamidi is currently a $\mathrm{PhD}$ holder and study in Solar Astrophysics specifically in radio astrophysics at the University of Malaya. Involve a project under the International Space Weather Initiative (ISWI) and also a lecturer in School of Physics and Material Science, at MARA University of Technology, Shah Alam Selangor.

N. N. M. Shariff: Her current research is more on sustainability; environmental aspect. She is looking forward for cross-field research, i.e. solar astrophysics, light pollution measurement (mapping) and religious studies.

C. Monstein is a senior Engineer at Institute of Astronomy, Wolfgang-Pauli-Strasse 27, Building HIT, Floor J, CH-8093 Zurich, Switzerland and one of the researchers who initiated the CALLISTO system around the world.

M. F. Ali is a Masters student at School of Physics and Material Science, at MARA University of Technology, Shah Alam Selangor.

W. N. A. Wan Zulkifli, is a final year student at School of Physics and Material Science, at MARA University of Technology, Shah Alam Selangor.

M. B. Ibrahim, is a final year student at School of Physics and Material Science, at MARA University of Technology, Shah Alam Selangor.

N. S. Arifin is a final year student at School of Physics and Material Science, at MARA University of Technology, Shah Alam Selangor.

N. A. Amran is a final year student at School of Physics and Material Science, at MARA University of Technology, Shah Alam Selangor. 


\section{References}

[1] A. O. Benz, Flare Observations, Living Rev. Solar Phys. 5 (2008).

[2] A. O. Benz, C. Monstein, H. Meyer, P. K. Manoharan, R. Ramesh, A. Altyntsev, A. Lara, J. Paez, Solar Phys. 55 (2004) 121-134.

[3] T. A. Chubb, Creplin, H. Friedman, J. Geophys. Res. 97 (1966).

[4] D. B.Melrose, ApJ 486 (1994).

[5] D. Herdiwijaya, S. Imelda, Jurnal Matematika dan Sains 11 (2006) 7.

[6] Benz A. O., C. Monstein, H. Meyer, CALLISTO, A New Concept for Solar Radio Spectrometers, Kluwer Academic Publishers, 2004.

[7] D. A. Guidice, Castelli J. P., Sol. Phys. 44 (1975).

[8] V. V. Grechnev, ApJ 566 (2002).

[9] V. Petrosian, High Energy Solar Physics, in: R. Ramaty, N. Mandzhavidze, X.-M. Hua (Eds.), Woodbury: AIP, 1996.

[10] G. W. Pneuman, Case of Hydromagnetic Resonance 2 (1967) 462-483.

[11] S. M. White, S. Krucker, R. P. Lin, Ap. J. Lett. 40 (2007).

[12] M. R. Kundu, Solar Radio Astronomy, John Wiley, 1965.

[13] Kundu Alissandrakis, Nature 257 (1975).

[14] A. V. R. Silva, H. Wang, D. E. Gary, Astrophys. J. 545 (2000).

[15] Z. S. Hamidi, N. Anim, N. N. M. Shariff, Z. Z. Abidin, Z. A. Ibrahim, C. Monstein, Dynamical structure of solar radio burst type III as evidence of energy of solar flares, in: R.Shukor (Ed.), PERFIK 2012, American Institute of Physics, Malaysia, 2013, pp. 11-15.

[16] Z. S. Hamidi, Z. Abidin, Z. Ibrahim, C. Monstein, N. Shariff, International Journal of Fundamental Physical Sciences 2 (2012) 32-34.

[17] Z. S. Hamidi, Z. Z. Abidin, Z. A. Ibrahim, N. N. M. Shariff, U. F. S. U. Ibrahim, R. Umar, Preliminary analysis of investigation Radio Frequency Interference (RFI) profile analysis at Universiti Teknologi MARA, IEEE, 2011, pp. 311-313.

[18] Z. S.Hamidi, U. F. S. U. Ibrahim, Z. Z. Abidin, Z. A. Ibrahim, N. N. M. Shariff, International Journal of Fundamental Physical Sciences 3 (2013) 20-23.

[19] Y. Liu, et .al., ApJ 691 (2009).

[20] Z. S. Hamidi, N. N. M. Shariff, C. Monstein, The International Journal of Engineering 1 (2012) 3 .

[21] I. H. Cairns, S. A. Knock, Predictions for dynamic spectra and source regions of type II radio bursts In the inhomogenous corona and solar wind Solar Physics 210 (2002) 419-430.

[22] Z. S. Hamidi, N. N. M. Shariff, International Letters of Chemistry, Physics and Astronomy 4 (2014) 8. 
[23] Z. S. Hamidi, N. N. M. Shariff, International Letters of Chemistry, Physics and Astronomy 5 (2014) 32-42.

[24] Z. S. Hamidi, N. N. M. Shariff, C. Monstein, W. N. A.W. Zulkifli, M. B. Ibrahim, N. S. Arifin, N. A. Amran, International Letters of Chemistry, Physics and Astronomy 8 (2014) $13-19$.

[25] Z. S. Hamidi, N. Shariff, Z. Abidin, Z. Ibrahim, C. Monstein, Middle-East Journal of Scientific Research 12 (2012) 6. 